\title{
Average surface roughness evaluation using 3-source photometric stereo technique
}

\author{
Thammarat Somthong and Qingping Yang* \\ College of Engineering, Design, and Physical Sciences, Brunel University London, Uxbridge, UK
}

Received: 21 March 2016 / Accepted: 12 October 2016

\begin{abstract}
This paper presents a technique for measuring surface roughness $(R a)$, using photometric stereo method. The method utilizes three or more images of the measured surface from the same viewpoint, taken sequentially under different illumination conditions. The scenes captured by the camera were reconstructed by the least square method to obtain surface normal from surface topography. Three-dimensional surface textural patterns were recovered from those surface normal. The system was validated with five standard surface roughness specimens. The $R a$ calculated from the recovered surface was compared with the values measured from contacting roughness measurement (stylus instrument). The best coefficient of the slant angles can also be determined through the linear regression. The experimental results indicate that the technique can be applied to well recover standard surface roughness.
\end{abstract}

Keywords: surface texture / photometric stereo / measurement / imaging techniques / metrology

\section{Introduction}

Computer graphics techniques have been extensively popular in the measurement system. The quality of components produced is of major concern in industrial manufacturing, and it is closely related to dimensional accuracy, form and surface finish. Surface topography, especially surface roughness, has an essential role to determine the functional performance of machine parts. The measurement of engineering surface roughness is becoming increasingly important. The traditional way of measuring surface topography is mechanical-most existing roughness standards are defined using stylus instruments that normally use a diamond stylus. Stylus can usually scratch the measured surface and may not be suitable when the measured surfaces are very soft, for example, in biological systems or polymers. In the last few decades, alternative methods rather than stylus have been developed. Those can be loosely divided into optical methods (such as vertical scanning interferometry) and non-optical methods (such as scanning tunneling microscopy) $[1,2]$.

Recently, photometric stereo method has been considered as a technique for roughness measurement. The photometric stereo is a method for evaluation of shape and reflectance of an object using three or more images under different lighting positions [3]. The method uses different lighting conditions to mainly measure the gradient field of the surface, which is calculated from an array of surface

\footnotetext{
* Corresponding author: Qingping.Yang@brunel.ac.uk
}

normal. The gradient field can be therefore considered to be the measurement results. The surface texture can be calculated from the obtained depth information [4-6].

In this paper, we consider the slant and tilt angles which are suitable for measuring different roughness ranging from $3.2 \mu \mathrm{m}$ to $50 \mu \mathrm{m}$. The $R a$ is then reconstructed by combining three images, each at a tilt angle with the object. By using the reconstructed $R a$ of the objects, parameters of a reflection model are estimated in a robust manner. The key point of the proposed method is that the linear regression technique was applied to find the best coefficient between image intensities and roughness.

\section{Operating principle}

\subsection{The reflectance model}

Intensity values are known from image sets that recorded a reflected radiance from the illuminated objects. There are many contributing factors relating to the reflected radiance when the incident light has constant direction and intensity, e.g. the physical properties and shape of the measured object. If the surfaces are rough enough so that all incident light beams are diffusively reflected to all directions, these surfaces are so-called lambertian surfaces [3]. According to the lambertian reflectance surface [3], this intensity is given by

$$
i=\rho k \cos \left(\theta_{i}\right)
$$

where $i$ is the emittance (reflected power per unit area) from the surface, $\rho$ is the albedo representing the amount of light reflected back from the surface, the intensity of light 


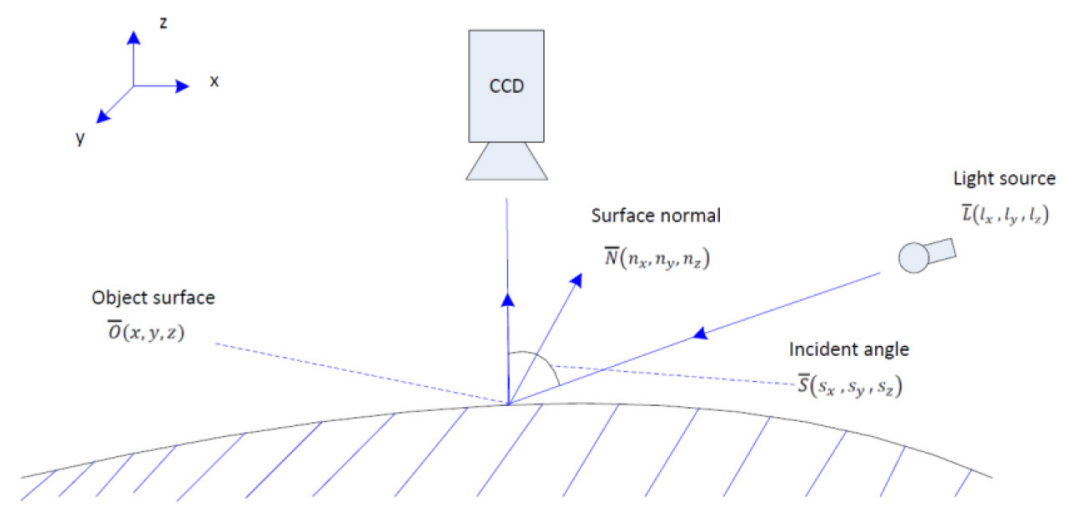

Fig. 1. Photometric stereo system.

source is represented by $k$ (incident power per unit area) and the angle between the light vector and the surface normal is represented by $\theta_{i}$.

\subsection{Photometric stereo (PS)}

The photometric stereo method was firstly proposed by Woodham in 1980. It is a method that calculates local surface reflection and orientation through the variation of incident light source, with several images taken from a constant viewing direction [3,4]. The basic idea of the PS method is to solve equation (2) for the unknown surface normal (Fig. 1).

When three images with the same view are taken under different lighting directions, three reflectance maps are provided for solving a linear system of three unknowns surface normal $\left(n_{x}, n_{y}, n_{z}\right)$. The cosine of the incident angle can also be denoted as dot product, as shown in equation (2), where $\vec{S}$ represents the unit vector of light source direction and the unit vector $\vec{N}$ represents the surface normal:

$$
I=\rho(\vec{S} \cdot \vec{N})
$$

In case of three images being utilized by the measurement system,

$$
\left[\begin{array}{l}
I_{1} \\
I_{2} \\
I_{3}
\end{array}\right]=\rho\left[\begin{array}{lll}
s_{x 1} & s_{y 1} & s_{1 z} \\
s_{x 2} & s_{y 2} & s_{2 z} \\
s_{x 3} & s_{y 3} & s_{3 z}
\end{array}\right]\left[\begin{array}{c}
n_{x} \\
n_{y} \\
n_{z}
\end{array}\right]
$$

In equation $(3),\left(I_{1}, I_{2}, I_{3}\right)$ represent the image intensity values at image location $(x, y)$. Unknown surface albedo is denoted by $\rho$. The unknown components of the surface normal is represented by $\left(n_{x}, n_{y}, n_{z}\right)$, and $\left(s_{x}, s_{y}, s_{z}\right)$ are the known components of light source vector, where numbers 1, 2 and 3 indicate light direction. To solve the unknown surface reflectance factor and surface normal, the following relation has been used:

$$
\vec{I}(x, y)=\rho(x, y)[S] \vec{N}(x, y) .
$$

To determine the surface normal, $N$, in equation (4), the following relation can be utilized from equation (5), where the lighting directions, $S$, must not be coplanar to a plane.

$$
\vec{N}(x, y)=\frac{1}{\rho(x, y)}[S]^{-1} \vec{I}(x, y)
$$

The least squares technique can be utilized to calculate Pseudo-Inverse and local surface gradients $p(x, y)$ and $q(x$, $y$ ). The local surface normal $\vec{N}(x, y)$ can also be calculated from the Pseudo-Inverse using equations (6)-(8) where $\vec{M}(x, y)=\left(m_{1}(x, y), m_{2}(x, y), m_{3}(x, y)\right)$.

$$
\begin{gathered}
\vec{M}(x, y)=\rho(x, y) \vec{N}(x, y)=\left([S]^{T}[S]\right)^{-1}[S]^{T} \vec{I}(x, y) \\
p(x, y)=\frac{m_{1}(x, y)}{m_{3}(x, y)}, q(x, y)=\frac{m_{2}(x, y)}{m_{3}(x, y)} \\
N(x, y)=\frac{[p(x, y), q(x, y), 1]^{T}}{\sqrt{p(x, y)^{2}+q(x, y)^{2}+1}} . \\
\rho(x, y)=\sqrt{m_{1}^{2}(x, y)+m_{2}^{2}(x, y)+m_{3}^{2}(x, y)}
\end{gathered}
$$

\section{Surface texture}

Surface measurement usually yields the information in two types, i.e. the roughness and the waviness. These parameters can be used to explain and predict the performance and problems of machine tools. Filtering techniques are applied to separate roughness and waviness from the surface topography $[7,8]$.

The surface profile parameters are defined by ISO 4287:1997 which encompasses five groups of texture parameters, including amplitude parameters (peak and valley), amplitude parameter (average of ordinates), spacing parameters, hybrid parameters, and curves and related parameters [9].

The surface roughness parameter used throughout this study is the arithmetical mean of the surface profile $(R a)$. This parameter is also the most widely used surface finish 


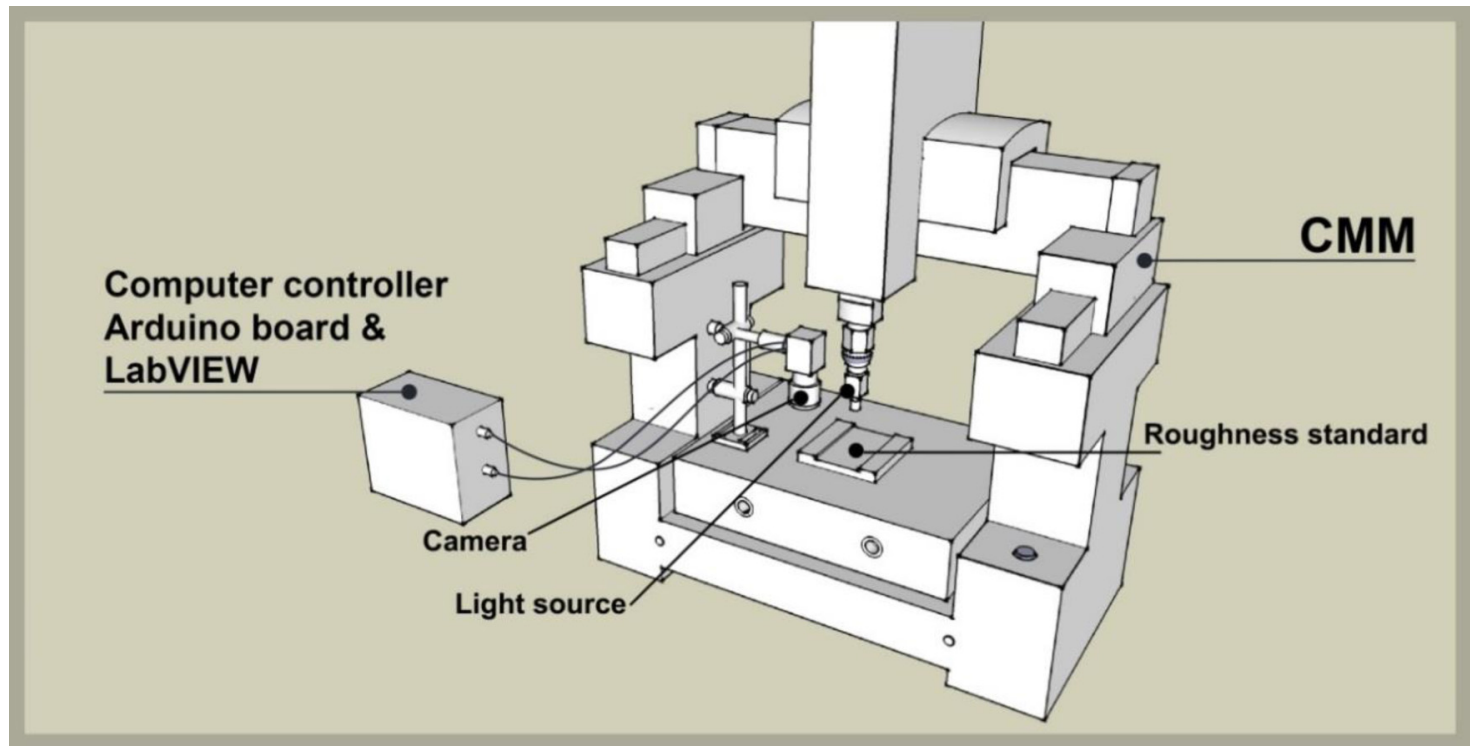

Fig. 2. The PS system on the CMM for measuring surface texture.

parameter by researchers and industries. $R a$ is the arithmetic average of the absolute value of the heights of roughness irregularities from the measured mean value $[7,10,11]$. It can be described in mathematical function as

$$
R_{a}=\frac{1}{N} \sum_{i=1}^{N}\left|Z_{i}\right|
$$

where $Z_{i}$ is the height of roughness irregularities from the mean value and $N$ is the number of measured points in a sampling length. $R a$ value can be calculated from the mean height $[9,10]$.

\section{System designs}

\subsection{Proposed photometric stereo system}

Figure 2 illustrates the PS system set up on a Coordinate Measuring Machine (CMM) for surface roughness measurement. The CMM is defined by ISO10360-1 as a measuring system with the means to move a probing system and the capability of determining spatial coordinates on a work piece surface [12,13]. The roughness standard was mounted at centre of the measuring table. The light source was assumed to be a point source with a constant incident illumination over the scene as shown in Figure 2. When images were captured from the system, the external lighting source was controlled. The measurements were performed in a dark room to prevent the effects of the ambient light.

The purpose of the experimental work was to investigate the practical application of PS techniques for measuring $R a$ from surface texture. The nominal values of standard surface roughness which included $3.2 \mu \mathrm{m}$, $6.3 \mu \mathrm{m}, 12.5 \mu \mathrm{m}, 25$ and $50 \mu \mathrm{m}$ were applied to validate the PS system [11]. Five differing surface roughness standards were viewed by a vision system. The light source was attached with the CMM's probe, which was able to move

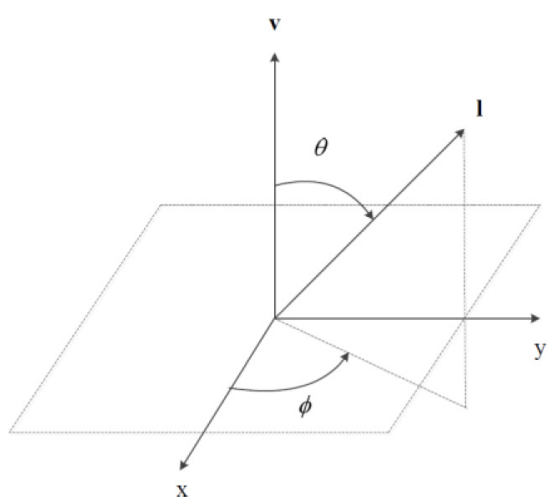

Fig. 3. Slant and tilt of a light source.

tilt and slant angles. The sensitivity of the technique to the slant angle was investigated. Three slant angles were examined: $\theta=37.5^{\circ}, 45^{\circ}$ and $52.5^{\circ}$. The optimal illumination configuration with regard to tilt angle is considered to be an intuitive result since the three vectors are equally spaced at $120^{\circ}$ apart.

\subsection{Optimal lighting position}

Spence and Chantler $[14,15]$ worked with three lights of equal slant and using numerical optimisation. They concluded that the normal are best recreated when the light sources are $120^{\circ}$ apart, and of slant $55^{\circ}$. This corresponds to orthogonal light directions and is in full agreement with the results derived from the theory presented here.

In Figure 3, the angle between light source (1) and viewing vector $(\mathbf{v})$ is the slant angle $(\theta)$. Tilt $(\varphi)$ is the angle between the projection of $\mathbf{l}$ onto the viewing plane $(x-y)$ and the $x$-axis. In this experiment, the slant angles $(\theta)$ were investigated at $37.5^{\circ}, 45^{\circ}$ and $52.5^{\circ}$ to find the optimum angle for measuring surface roughness standard. The slant angle was held constant whilst the tilt angle was swept 


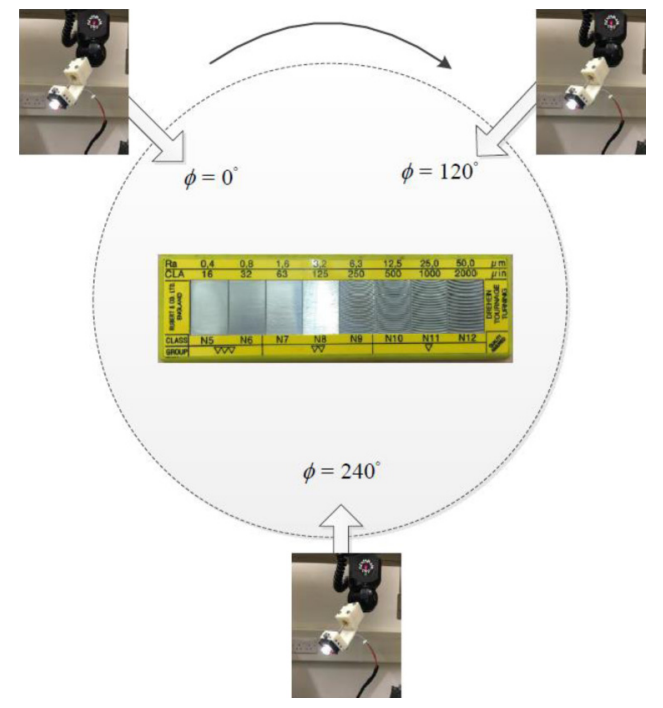

Fig. 4. Illumination conditions for the tilt angle experiments.

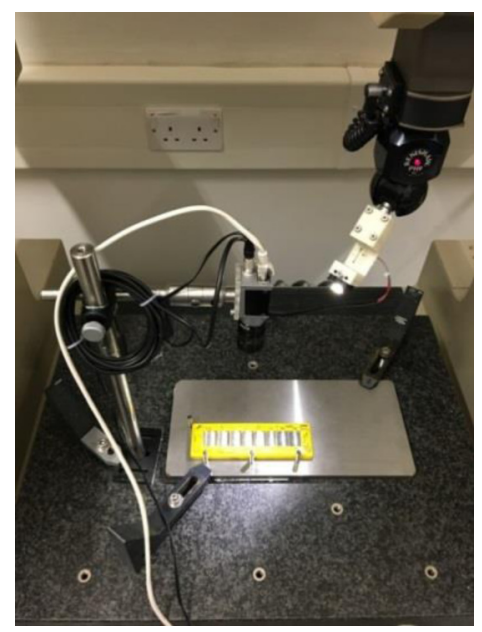

Fig. 5. Light source.

through a complete rotation in $120^{\circ}$ intervals. Three images were captured at $120^{\circ}$ intervals over a complete rotation at each of the three slant angles (Fig. 4).

\subsection{Light source}

A neutral white LED was used as the light source. It is properly designed for industrial lighting and provides high illumination intensity 230 lumens at $700 \mathrm{~mA}$ driver. A narrow beam $\left(17.7^{\circ}\right)$ optical lens was used for increasing the efficiency of light source. The light source and captured images were controlled by the Arduino board and LabVIEW software shown in Figures 5 and 6 . The angle of light was moveable via the movement of the CMM to find the suitable tilt and slants angles. In terms of tilt angles, the samples were illuminated at angles $0^{\circ}$, $120^{\circ}$, and $240^{\circ}$. With regard to the slant angle, the measurement results were found dependent on the characteristics of the material and texture of the specimen. In this study, we found that the optimal slant angle for measuring the roughness standard between $3.2 \mu \mathrm{m}$ and $50 \mu \mathrm{m}$ was $45^{\circ}$.

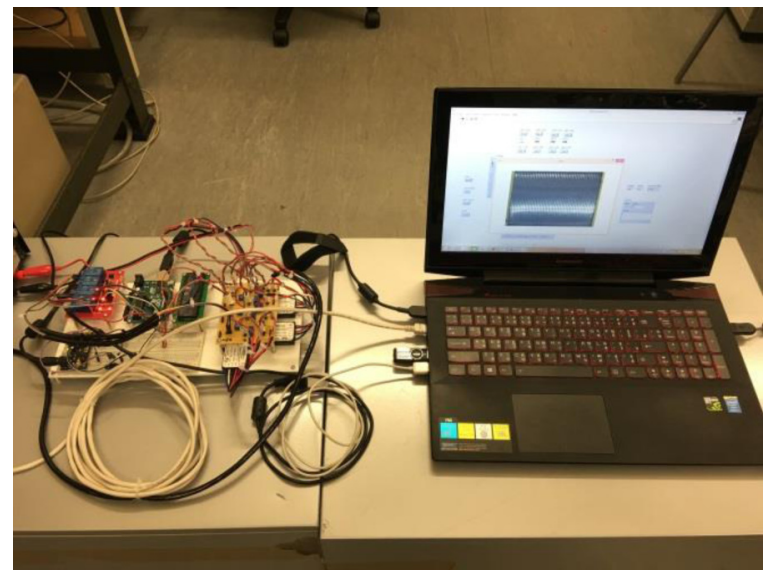

Fig. 6. Arduino board and LabVIEW software.

\subsection{Measurement method}

Using the PS method for measuring surface texture can be written as block diagrams in Figure 7.

The block diagram shows the six steps for measurement method. The light source is used to create a stable and wellcontrolled illumination condition. The images with dimensional size $659 \times 494$ pixels are captured from the Basler Ace Digital Gigabit Ethernet camera with a $25 \times$ optical zoom lens. Those images acquired from each tilt angle are taken precisely at position determined by the CMM's movement. In case of mark images, the area of interest is selected by Matlab programming. Data acquisition process is done by using Arduino board controlled by LabVIEW software. Gradient space plots are generated and analysed for all standard surface roughness. The surface normal is therefore calculated by the least squares technique following by equation (6). Therefore, accurate surface reconstruction results can be achieved. Twelve profiles from the measured surfaces are then chosen for intensity calculation and compared with the roughness standards.

\section{Experimental results}

\subsection{Standard roughness calibration}

According to ISO 5436-1 [10], the surface roughness standards can be separated by purposes and metrological characteristics and are used for five types of measurements including type A, B, C, D and E. The surface roughness standard type $\mathrm{D}$ characterized $R a$ was utilized as the reference of our system (Fig. 8).

Surface roughness standards used in the experiment comprised $R a$ values of $3.2,6.3,12.5,25$ and $50 \mu \mathrm{m}$, respectively. They were calibrated with the standard contact method, using a stylus instrument (Surfcorder ET4000A) to determine the average roughness values and measurement uncertainties. The uncertainty of measurement of the stylus instrument was stated in the calibration certificate as $\sqrt{\left(10^{2}\right)+\left(12^{2} \times R_{a}\right)} \mathrm{nm}$, where $R_{a}$ is the 


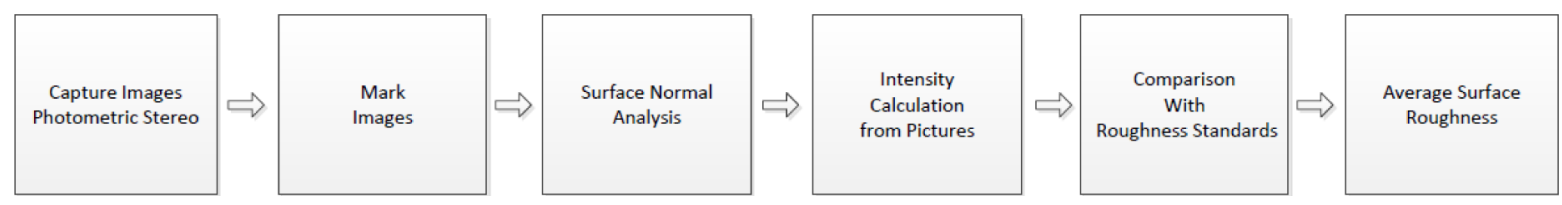

Fig. 7. Inspection strategy.

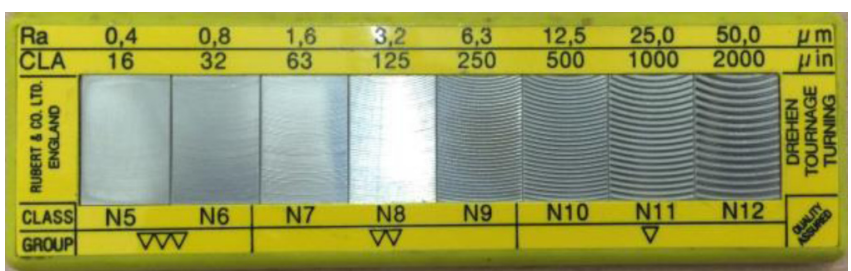

Fig. 8. Surface roughness standard Type D following ISO 5436-1.

measured roughness value in $\mu \mathrm{m}$. The measurement results were shown in Table 1 and the roughness standard profiles measured from the stylus instrument were presented in Figure 9.

The original images of the surface roughness standard under three light directions are shown in Figure 10. The idealised simulated surface roughness standard from the PS system is shown in Figure 11. The 3D recovery of the surface roughness standard measured using from the PS system is illustrated in Figure 12.

\subsection{Computing average surface roughness using Photometric stereo method}

The sample profiles are captured, with its centre coincides with the image centre. Each image acquired from the camera covers an area of specimen around $512 \times 340 \mathrm{~mm}$. For measuring $R a$ profiles according to the ISO 4288:1996 [11], the 12 lines selected from the recovered three-dimensional profiles are calculated by equation (10).
Table 1. Surface roughness standards measured by the stylus instrument.

\begin{tabular}{lll}
\hline $\begin{array}{l}\text { Nominal } \\
\text { value }(\mu \mathrm{m})\end{array}$ & $\begin{array}{l}\text { Stylus } \\
\text { instrument }(\mu \mathrm{m})\end{array}$ & $\begin{array}{l}\text { Standard } \\
\text { deviation }(\mu \mathrm{m})\end{array}$ \\
\hline 3.2 & 3.13 & 0.03 \\
6.3 & 5.79 & 0.03 \\
12.5 & 14.36 & 0.03 \\
25 & 31.67 & 0.58 \\
50 & 60.45 & 0.14 \\
\hline
\end{tabular}

The polynomial curve fitting is utilized to find reference line as shown in Figure 13. The $R a$ profile measured from the PS method is illustrated in Figure 14. All measurements performed at the metrology laboratory of Brunel University London were controlled environmental conditions, especially in temperature and vibration.

\subsection{Measurement of roughness with high pass filters}

Roughness is mathematically described as a high frequency deviation from the ideal surface[16]. To determine the roughness of a surface, form and waviness must be separated from the raw measured data. A high pass filter has been applied to eliminate them. For further examinations the weighting function for the phase correct filter corresponding to the equation of the Gaussian density function would be utilized. The definition of weighting

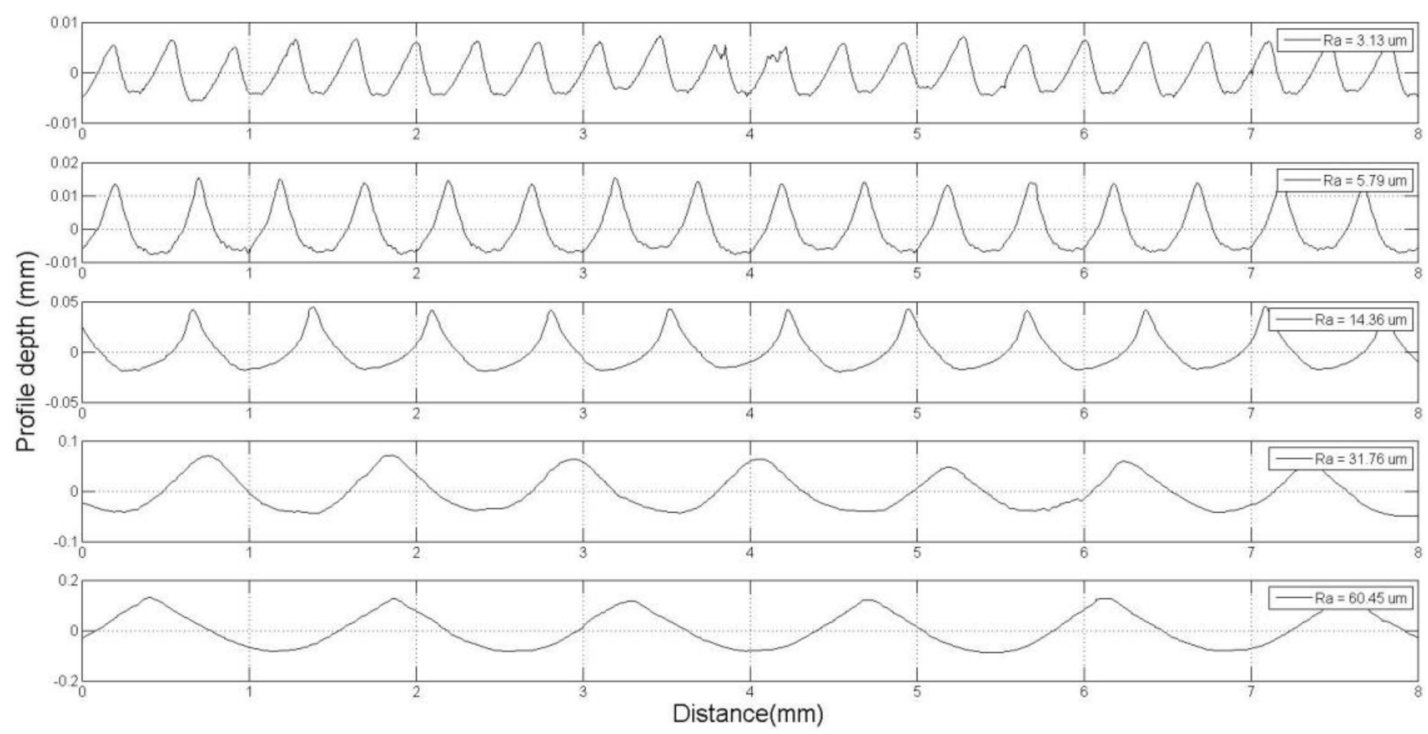

Fig. 9. Surface roughness standard profiles measured from the stylus instrument. 
function is explained in the ISO 16610-61:2015 standard [17]. Its weight function is given by

$$
h(x)=\frac{1}{\alpha \lambda_{\text {co }}} \exp \left[-\pi\left(\frac{x}{\alpha \lambda_{\text {co }}}\right)^{2}\right],
$$

where $x$ is the surface roughness profiles determined in $\mathrm{X}$ direction, $\lambda_{\text {co }}$ is the cut-off wavelength and the constant $\alpha$ is given by

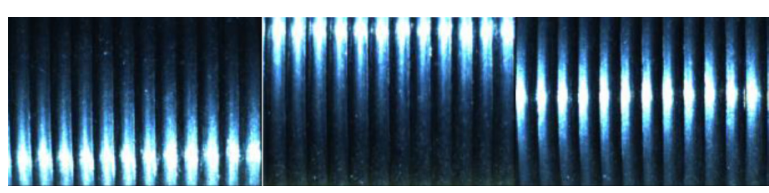

Fig. 10. Three images of roughness standard illuminated from a tilt angle of $0^{\circ}, 120^{\circ}$ and $240^{\circ}$.

$$
\alpha=\sqrt{\frac{\ln 2}{\pi}} .
$$

The different weight function results would be varied with the $\lambda_{\text {co. }}$ In the value of surface roughness standards, the $\lambda_{\text {co }}=2$ and $\lambda_{\text {co }}=6$ were created as cut-off wavelength as illustrated in Figure 15.

To obtain the transfer function $H(\lambda)$ of the respective filter, a Fourier transformation of $h(x)$ is necessary.

$$
\begin{aligned}
H(\lambda) & =\int_{-\infty}^{\infty} h(x) \exp \left[-i \frac{2 \pi}{\lambda} x\right] d x \\
& =\exp \left[-\pi\left(\frac{\alpha \lambda_{\mathrm{co}}^{2}}{\lambda}\right)\right] .
\end{aligned}
$$

The Gaussian transfer function created from equation (13) is the typical characteristic of a low pass filter. In term of calculating high pass filter, the transmission characteristic of the short wave component is obtained by $1-H(\lambda)$.

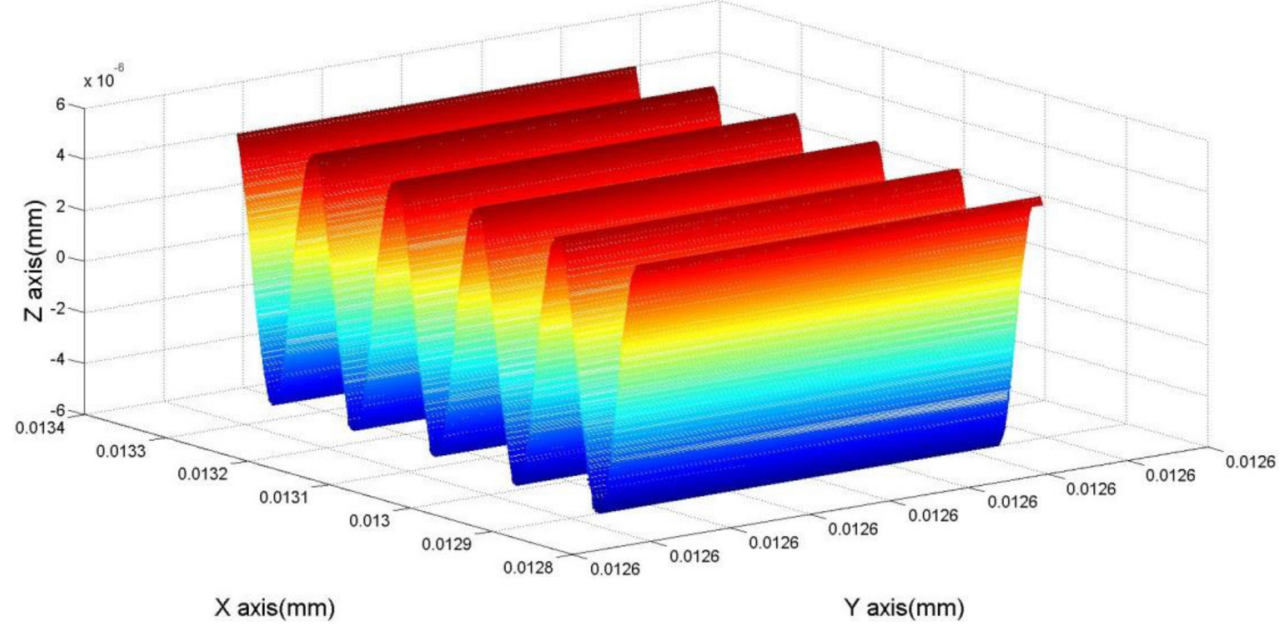

Fig. 11. Idealised simulated surface roughness standard.

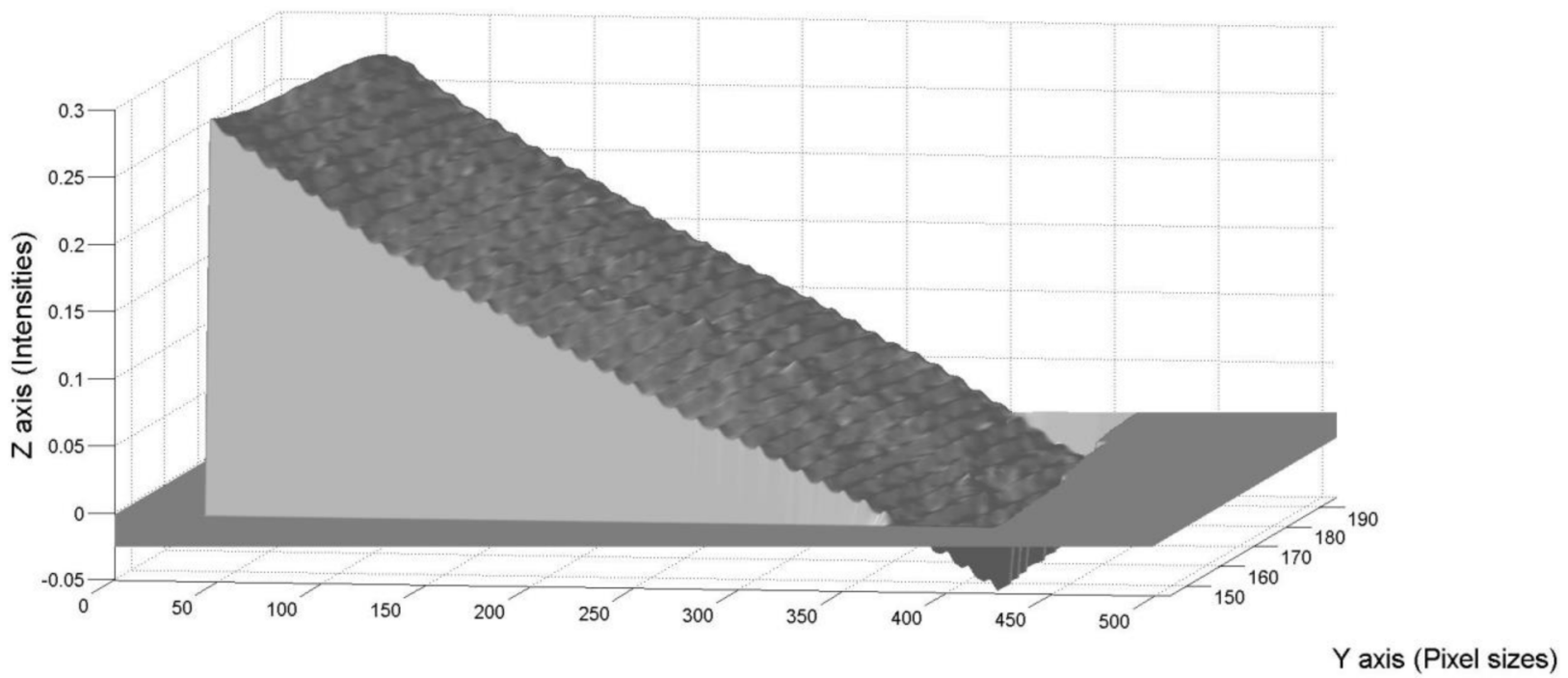

$X$ axis (Pixel sizes)

Fig. 12. Recovered three dimensional surface roughness standard at $6.3 \mu \mathrm{m}$. 


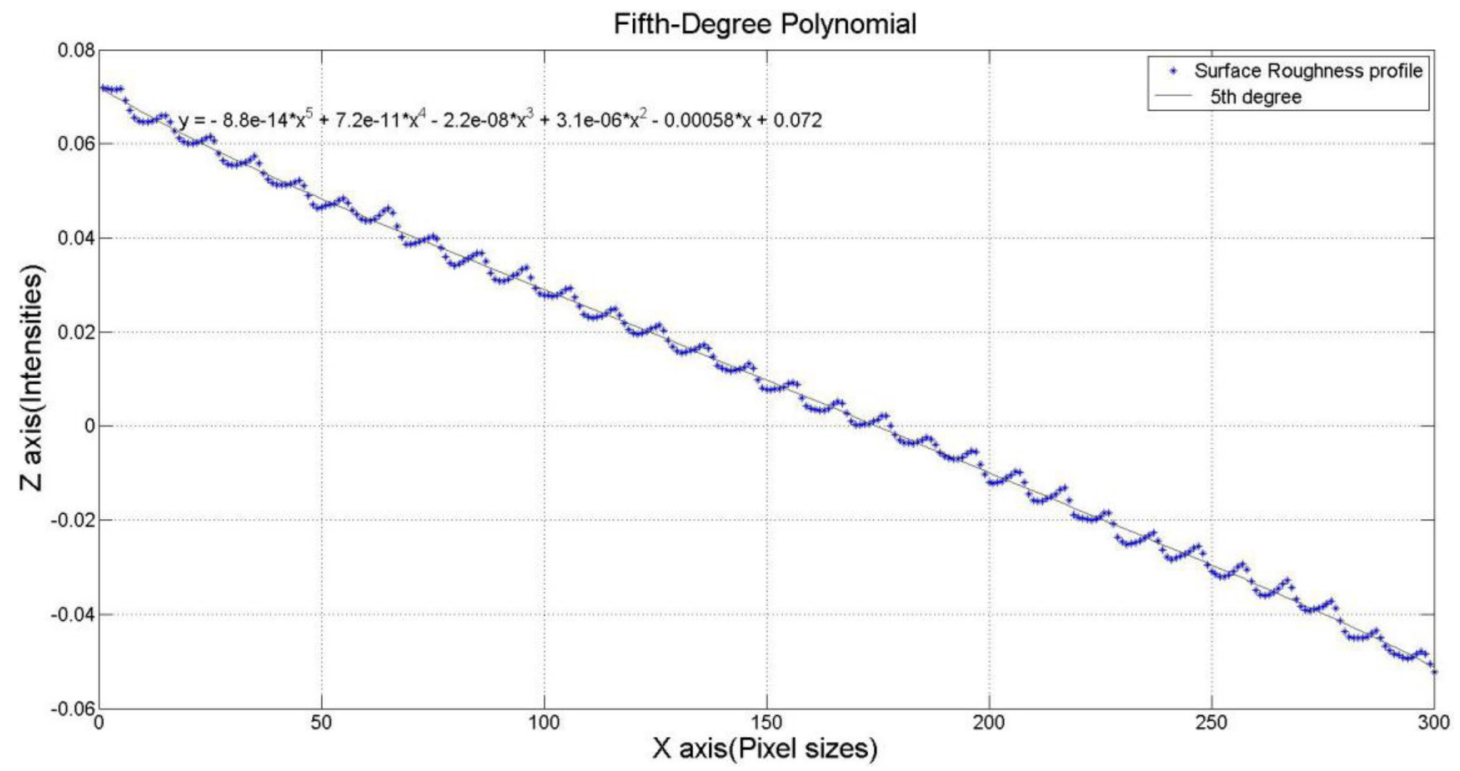

Fig. 13. The polynomial curve fitting.

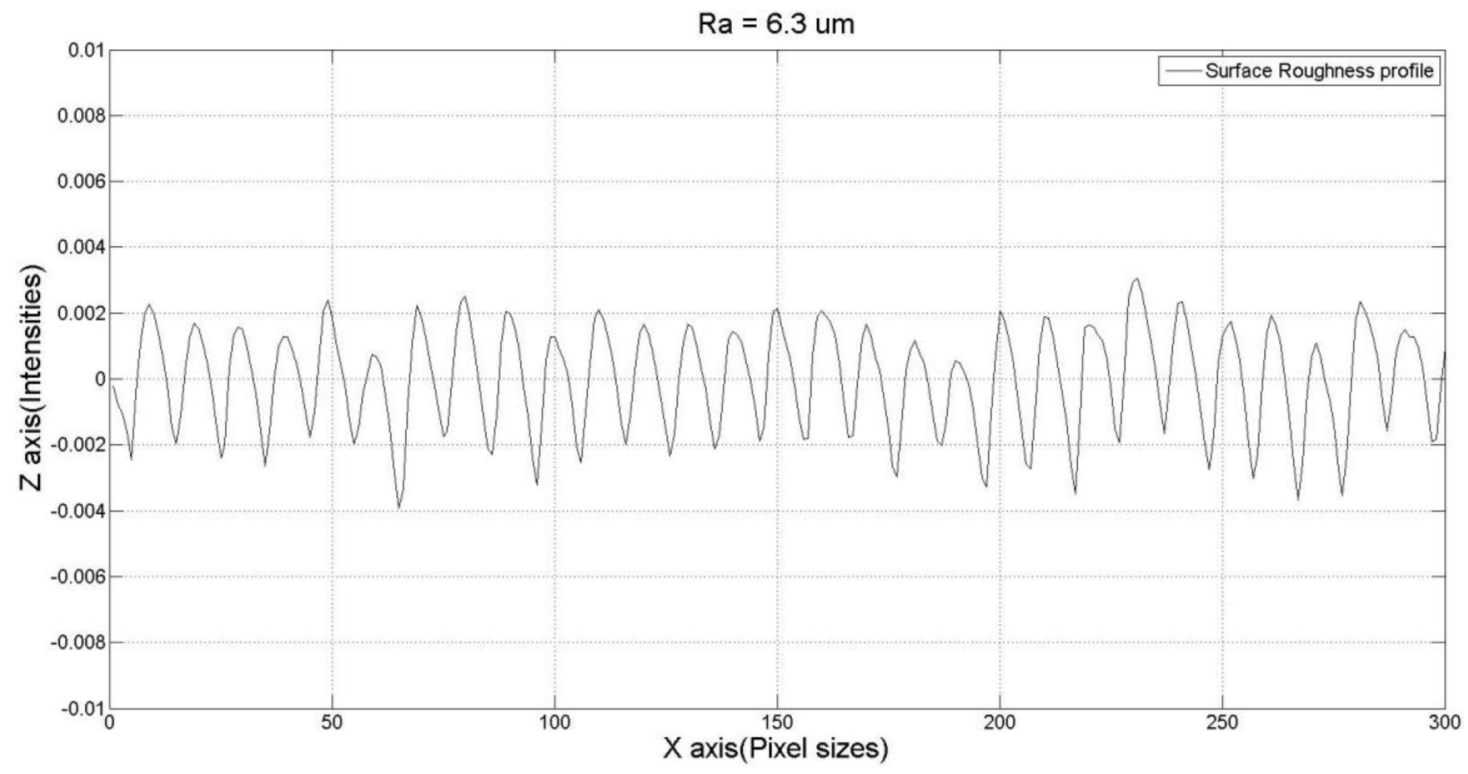

Fig. 14. Surface roughness standard profiles $6.3 \mu \mathrm{m}$ after using the polynomial curve fitting.

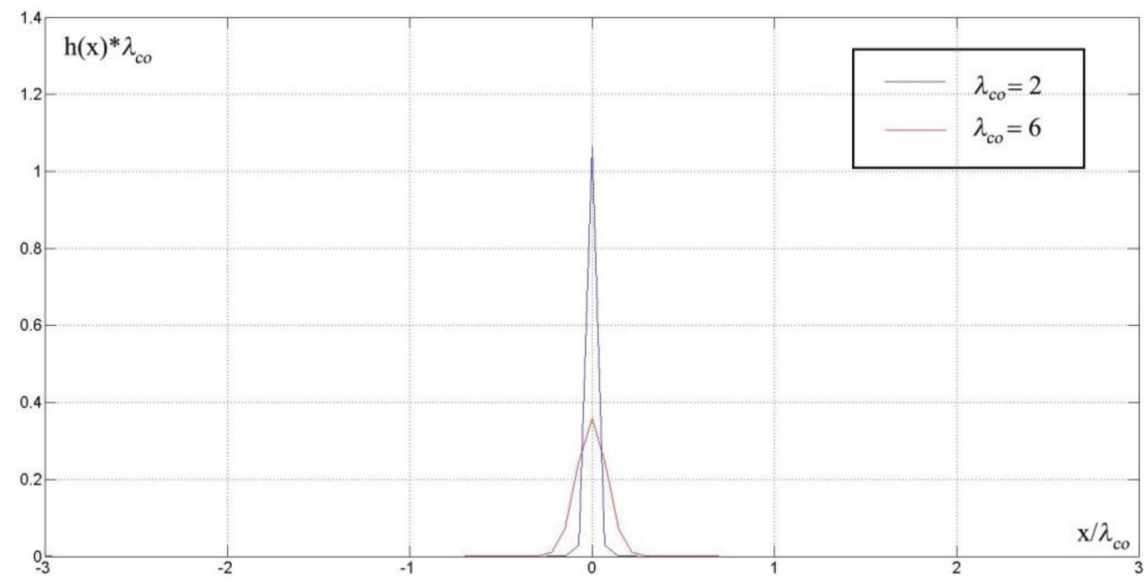

Fig. 15. Gaussian density function at $\lambda_{\text {co }}=2$ and $\lambda_{\text {co }}=6$. 


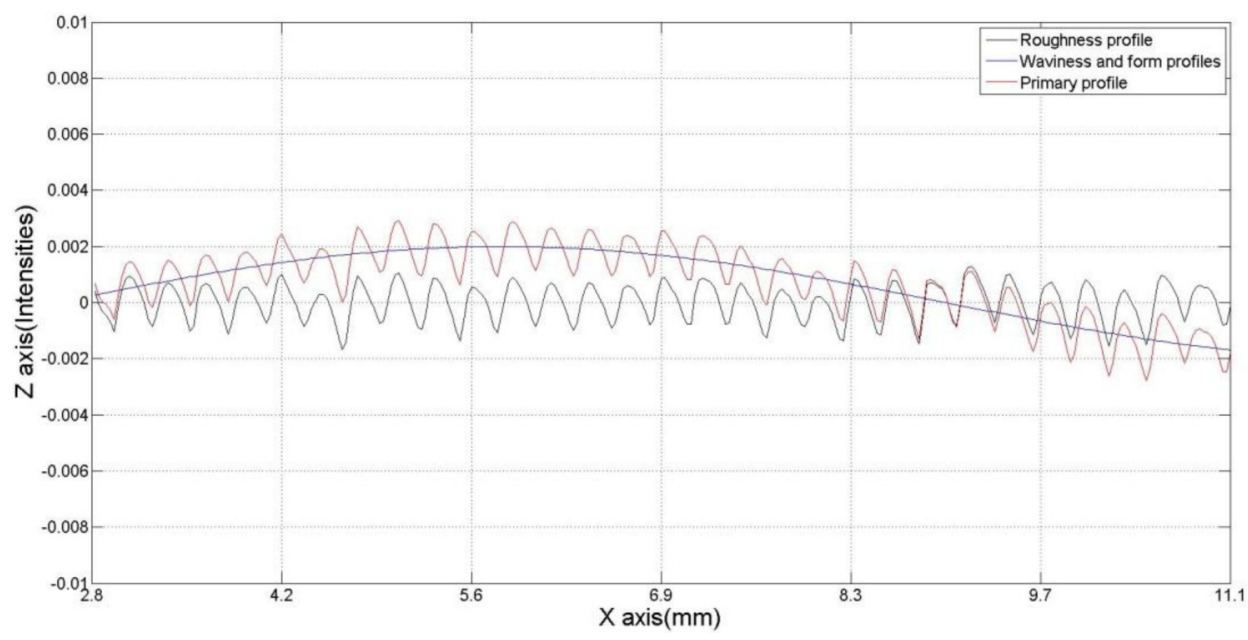

Fig. 16. Surface roughness standard profiles $6.3 \mu \mathrm{m}$ separated from primary profile by high pass filter.

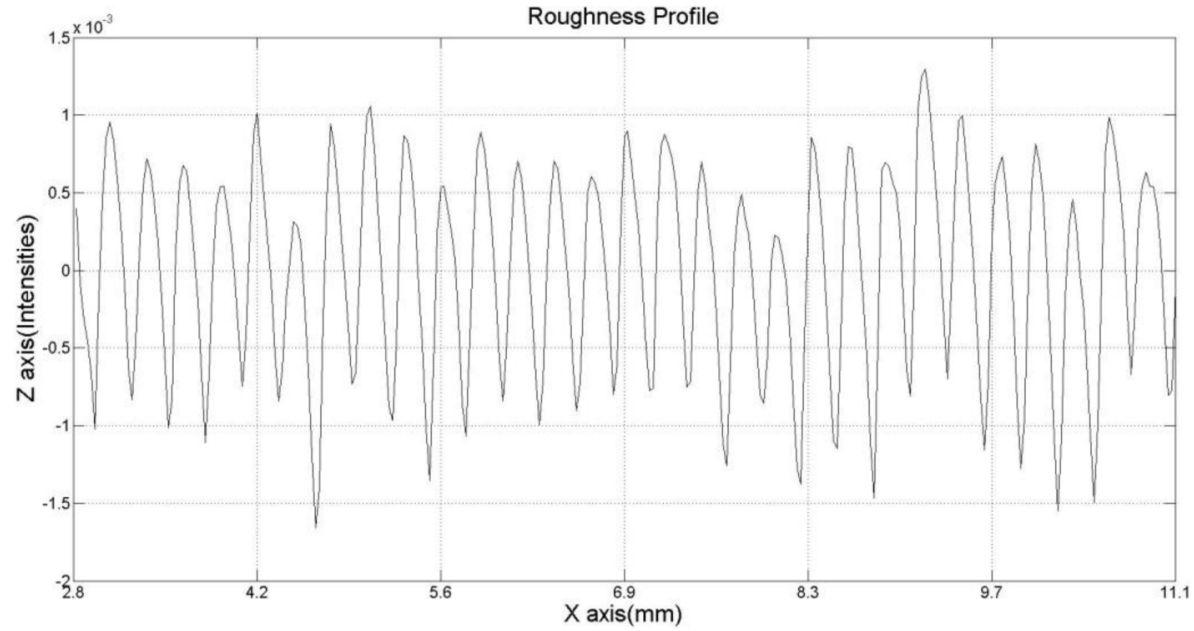

Fig. 17. Surface roughness standard profiles $6.3 \mu \mathrm{m}$ measured from the PS method after filtering.

The Gaussian transfer functions applied on surface roughness standard profiles before filtering are able to separate roughness profile from form and waviness profiles illustrated in Figure 16. In addition, the $x$ axis of measurement results in Figures 16 and 17 have been converted from pixel sizes to length measurement in $\mathrm{mm}$ unit based on the camera calibration. To reduce the statistical error of a measurement, it is necessary to perform several averages per position. There are a few points which show a high deviation from surface model. It can be then expected that the standard deviation would be very small. The result of $R a$ profiles measured from the PS method after high pass filter is shown in Table 2.

For each value of surface texture measured from the PS method, the surface roughness standard values are solved by the system of linear equation $[18,19]$. The solution for the coefficients is given by

$$
A X=B
$$

$A$ and $B$ are metrics whose elements are known, and $X$ is a column vector whose elements are unknown. The equation can be drawn from the measurement system as follows

$$
\begin{gathered}
X=\left[\begin{array}{l}
m \\
b
\end{array}\right], \\
A=\left[\begin{array}{cc}
x_{1} & 1 \\
x_{2} & 1 \\
\vdots & \vdots \\
x_{n} & 1
\end{array}\right], \\
B=\left[\begin{array}{c}
y_{1} \\
y_{2} \\
\vdots \\
y_{n}
\end{array}\right],
\end{gathered}
$$

where $x_{1}, x_{2}, \ldots, x_{n}$ are the data determined from the PS method, and $y_{1}, y_{2}, \ldots, y_{n}$ are the surface roughness standard values determined from the stylus instrument. Slope or gradient of line are shown by constant $m$, and $b$ is a point at which the line crosses the $y$-axis. The best 
Table 2. The measurement results of $R a$ determined from the PS method under different slant angles.

\begin{tabular}{ccll}
\hline Slant & $\begin{array}{l}\text { Standard } \\
\text { roughness } \\
\text { nominal value }(\mu \mathrm{m})\end{array}$ & $\begin{array}{l}\text { PS method } \\
\text { average } \\
\text { value }\end{array}$ & $\begin{array}{l}\text { Standard } \\
\text { deviation }\end{array}$ \\
\hline & 3.2 & 0.00027 & 0.000001 \\
$37.5^{\circ}$ & 12.5 & 0.00041 & 0.000002 \\
& 25 & 0.00051 & 0.000001 \\
& 50 & 0.00096 & 0.000002 \\
& 3.2 & 0.0011 & 0.000002 \\
$45^{\circ}$ & 12.5 & 0.00020 & 0.000001 \\
& 25 & 0.00025 & 0.000002 \\
& 50 & 0.00030 & 0.000002 \\
& 3.2 & 0.00049 & 0.000001 \\
& 6.3 & 0.00065 & 0.000002 \\
$52.5^{\circ}$ & 12.5 & 0.00013 & 0.000001 \\
& 25 & 0.00022 & 0.000001 \\
& 50 & 0.00027 & 0.000002 \\
& & 0.00040 & 0.000002 \\
& & 0.00045 & 0.000002 \\
\hline
\end{tabular}

Table 3. Linear regression between surface roughness standard determined from the stylus instrument and PS technique results under different slant angles.

\begin{tabular}{cll}
\hline $\begin{array}{l}\text { Slant } \\
\text { angle }(q)\end{array}$ & Linear regression model & $\begin{array}{l}\text { Coefficient of } \\
\text { determination }\end{array}$ \\
\hline $37.5^{\circ}$ & $R a_{\text {std }}=62406 R a_{\mathrm{PS}}-17.42$ & 0.90 \\
$45^{\circ}$ & $R a_{\text {std }}=126195 R a_{\mathrm{PS}}-24.78$ & 0.98 \\
$52.5^{\circ}$ & $R a_{\text {std }}=169334 R a_{\mathrm{PS}}-26.84$ & 0.85 \\
\hline
\end{tabular}

coefficient was found at $45^{\circ}$ of slant angle. The linear regression model at $45^{\circ}$ of slant angle can work well for recovering three dimensional surface roughness standards. The measurement results are summarized in Table 3 as the coefficients of determination between the estimated $R a$ by the PS system against surface roughness determined from the stylus instrument.

$R a_{\mathrm{PS}}$ is roughness standard profiles averaged from 12 lines from image measurement system, and $R a_{\text {std }}$ is value measured by the stylus instrument. Five values of surface roughness standards were utilized for evaluation of the PS system. The best coefficient was found at $45^{\circ}$ of slant angle. This equation was offered for roughness average estimation form recovered surface.

\section{Conclusion}

The experimental results have shown that the new approach to surface textural measurement using photometric stereo method and CMM is capable of measuring surface roughness of work pieces. Moreover, the errors in the light source positioning can be significantly reduced by the CMM, and the PS system can improve the measurement accuracy. The surface roughness preliminarily tested ranges from 3.2 to $50 \mu \mathrm{m}(R a)$ and the measurement result can be correctly filtered to remove low frequency signals. The results of slant angle experiment can be easily used to optimize the system of roughness measurement. The work is potentially useful for accuracy improvement for PS method based on metrology and reverse engineering. In addition, the work can be well applied to measure the $R a$ for on-line industrial measurement. The further investigation includes characterization of the measurement performance and evaluation of measurement uncertainty of the PS method.

\section{References}

1. S. Damodarasamy, S. Raman, Texture analysis with computer vision, Comput. Ind. 16, 25-34 (1991)

2. G.A. Al-Kindi, R.M. Baul, K.F. Gill, An application of machine vision in the automated inspection of engineering surfaces, Int. J. Prod. Res. 30, 241-253 (1992)

3. R.J. Woodham, Determining surface curvature with photometric stereo, IEEE Int. Conf. Robot. Autom. 1, 36-42 (1989)

4. M.L. Smith, The analysis of surface texture using photometric stereo acquisition and gradient space domain mapping, Image Vis. Comput. 17, 1009-1019 (1999)

5. M.L. Smith, G. Smith, T. Hill, Gradient space analysis of surface defects using a photometric stereo derived bump map, Image Vis. Comput. 17, 321-332 (1999)

6. E. North Coleman, R. Jain, Obtaining 3-dimensional shape of textured and specular surfaces using four-source photometry, Comput. Graph. Image Process. 18, 309-328 (1982)

7. D. Whitehouse, Surface and Their Measurement (Hermes Penton, London, 2002), p. 10

8. R. Leach, Good Practice Guide No. 37 The Measurement of Surface Texture using Stylus Instruments (National Physical Laboratory, United Kingdom, 2001)

9. ISO 4287:1997, Geometrical Product Specifications (GPS) Surface texture: Profile method - Terms, definitions and surface texture parameters (International Organization for Standardization, Geneva, Switzerland, 1997), pp. 10-16

10. ISO 4288:1996, Geometrical Product Specifications (GPS) Surface texture: Profile method - Rules and procedures for the assessment of surface texture (International Organization for Standardization, Geneva, Switzerland, 1996), p. 2

11. ISO 5436:2000, Geometrical Product Specifications (GPS) Surface texture: Profile method; Part 1: Material measures (International Organization for Standardization, Geneva, Switzerland, 2000), p. 7

12. S. Hammad Mian, A. Al-Ahmari, New developments in coordinate measuring machines for manufacturing industries, Int. J. Metrol. Qual. Eng. 5, 101 (2014)

13. ISO 10360-1, Geometrical Product Specification (SPC). Acceptance and reverification tests for coordinate measuring machines (CMM). Part 1: Vocabulary (International Organization for Standardization, Geneva, Switzerland, 2000)

14. A. Spence, M. Chantler, Optimal illumination for threeimage photometric stereo acquisition of texture, in Proceedings of the 3rd International Workshop on Texture Analysis and Synthesis (2003), pp. 89-94 
15. O. Drbohlav, M. Chantler, On optimal light configurations in photometric stereo, in Proceedings of the 10th IEEE International Conference on Computer Vision (2005), pp. 1707-1712

16. J. Lieb, H. Biskup, S. Draxinger, R. Rascher, C. Wunsche, Process development for the reproducible roughness measurement of optical surfaces using white light interferometry, Int. J. Metrol. Qual. Eng. 5, 104 (2014)
17. ISO 16610-61:2015, Geometrical Product Specifications (GPS) - Filtration Part 61: Linear areal filters - Gaussian filters (BSI Standards Limited, UK, 2015)

18. W.J. Palm III, Introduction to MATLAB 7 for Engineers (McGraw-Hill, New York, 2005), p. 312

19. S.T. Karris, Numerical Analysis Using MATLAB and Excel (Orchard Publication, Fremont, CA, 2007), pp. 4-25

Cite this article as: Thammarat Somthong, Qingping Yang, Average surface roughness evaluation using 3-source photometric stereo technique, Int. J. Metrol. Qual. Eng. 7, 406 (2016) 